\title{
Esthetic evaluation of early rhinoplasty with complete unilateral cleft lip repair after nasoalveolar molding
}

\author{
Mohammed Ellithy ${ }^{\mathrm{a}}$, Osama Alshahat ${ }^{\mathrm{b}}$, Refaat Ibrahima ${ }^{\mathrm{a}}$, Wael Elmohandes ${ }^{\mathrm{c}}$, Sadek Abdelrahman ${ }^{\mathrm{d}}$ \\ ${ }^{a}$ Pediatric surgery department, Al-Azhar university. ${ }^{b}$ Plastic surgery department Al-Azhar university. ${ }^{c}$ Dental \\ surgery, Al-Azhar university. ${ }^{\mathrm{d}}$ General surgery department, Al-Azhar university.
}

Corresponding author; dr. Refaat Ibrahim. Email: dr.refaatbadawy@ hotmail.com, Mobile: 01001567863

\begin{abstract}
:
Background: The aesthetic outcome of primary rhinoplasty with complete unilateral cleft lip repair is a challenging due to nasal deformities, tissue deficiencies and alveolar ridge displacement. The aim of this study is aesthetic evaluation of primary rhinoplasty with complete unilateral cleft lip repair after nasoalveolar molding (NAM). Patient and methods: The study was applied on 30 patients with complete unilateral cleft lip, mean age at presentation was 89.17 73.02 days (range 15-260 days). Presurgical nasoalveolar molding (PNAM) was done for all patients with mean duration of molding 78.48 5.635 days (range 66-87 days). Measurements of nose and alveolar defect were taken by caliper before application of the device and after application of the device and after one month of operation and compared with each other's. All patients were subjected to lip repair with Millard technique with primary closed rhinoplasty. Primary rhinoplasty with cleft lip repair becomes easier since NAM diminishes the tension over the soft tissues and reduces severity of deformity in the lower lateral alar cartilages. Postoperative nasal stent has a great role in improve the esthetic outcome of primary rhinoplasty. Results: There were significant improvement of nasal symmetry and reduction of alveolar gap width (AGW) after use of NAM. Conclusion: This study suggests that PNAM within 15 days post-delivery is effective at reducing alveolar gap width, alignment of cleft alveolar segments and improvement of nasal symmetry as long as the parents cooperate in placing and maintaining the NAM device in the newborn's mouth and nose.
\end{abstract}

Keywords: Nasoalveolar molding, Unilateral cleft lip, Esthetic evaluation, Early rhinoplasty, Primary closed rhinoplasty.

\section{Introduction}

Cleft lip with or without cleft palate is the most common congenital malformation of the head and neck. The impact on quality of life for the child and the family can be severe, particularly in unsuspecting families. Emotional and psychological needs must be recognized and addressed, in for all those involved with the patient ${ }^{(\mathbf{1})}$. It is reported that the cleft lip and palate has significant variation depending upon geographical location, racial and ethnic background and socio economic status ${ }^{(2)}$. They may vary from minor notching of lip or bifid uvula to complete unilateral or bilateral cleft of the lip and palate ${ }^{(3)}$. The overall prevalence of orofacial cleft OFC is estimated to be approximately 1 in 700 live births, accounting for nearly one half of all craniofacial anomalies. As reported by the World Health Organization (WHO), the prevalence at birth of OFC varies worldwide, ranging 3.4-22.9 per 10,000 births for cleft lip, with or without cleft palate $(\mathrm{CL} / \mathrm{P})$, and $1.3-25.3$ per 10,000 births for cleft palate alone (CPO). The risk factors for CL/P and $\mathrm{CP}$ include family history, maternal nutrition, and exposure to teratogenic agents ${ }^{(4)}$. The cleft lip is typically repaired at 3 to 5 months of age, but may be later if Presurgical nasoalveolar molding (PNAM) is chosen. PNAM involves the repositioning of the alveolar segments and deformed lower lateral cartilages by active molding in the neonatal period prior to the primary cheiloplasty. The alveolar segments, premaxilla, and surrounding soft tissue gradually shift to their normal anatomical positions.

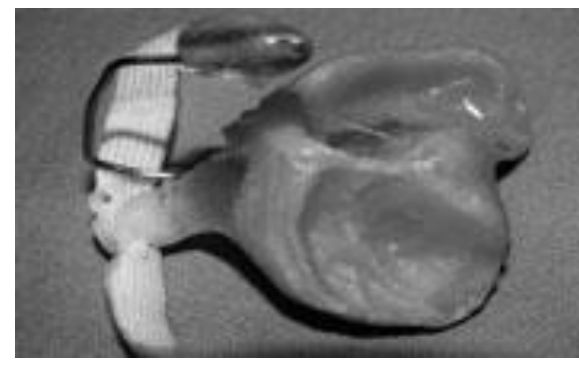

Figure 1: Device for presurgical nasoalveolar molding. 
The principle of NAM is based on the breakdown of the intercellular matrix of nasal cartilage due to the abundance of hyaluronic acid during infant's first 6-8 weeks. During this period, there are high levels of maternal estrogen in fetal circulation, which triggers an increase in hyaluronic acid. Furthermore, according to Hamrik's chondral modeling hypothesis, NAM is thought to simulate immature nasal chondorblasts producing the interstitial expansion, which in turn improve nasal morphology ${ }^{(5)}$. However, not all cleft centers apply this treatment to their patients because the effect of nasoalveolar molding is still the subject of debate. In addition, the different techniques of NAM, starting age, treatment duration, timing of surgery, and surgical technique results are limited scientific evidence regarding the long-term effect of the good nasal contour. Primary rhinoplasty is defined as nasal surgery at the time of primary cleft lip repair. Which should be considered a part of the overall cleft pathology. The goal of primary rhinoplasty for both the unilateral and bilateral deformity is to provide better contour, symmetry, and projection of the nasal tip by releasing, undermining, and repositioning the lower lateral cartilages $^{(6)}$. A successful orofacial reconstruction must include symmetry of lip height and a Cupid's bow, creation of natural philtral columns and dimple, continuity of a well aligned white roll and vermilion, and functional reconstruction of the orbicularis oris. At the conclusion of the rhinoplasty, the cleft side lower lateral cartilage ideally has normal projection without buckling or hooding. The aim of this study is aesthetic evaluation of primary rhinoplasty with complete unilateral cleft lip repair after nasoalveolar molding (NAM).

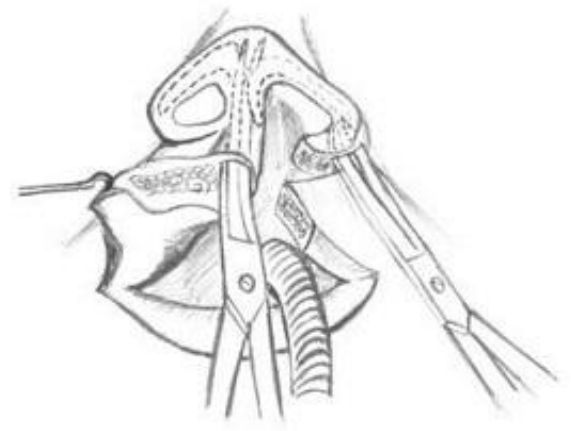

Figure 2: Dissection for access to crura.

\section{Patient and methods}

This is a prospective study of outcomes of presurgical nasoalveolar molding in correction of nasal deformities with cleft lip repair. It is conducted at Pediatric Surgery Department, AlAzhar University Hospitals over a period of two years. It is done on 30 children with cleft lip and nose. All patients were submitted to PNAM before primary rhinoplasty and cleft lip repair. Informed consent is obtained from the parents.

Inclusion criteria: 1) Patients with complete unilateral cleft lip. 2) Age from 15 days to 1 year.

Exclusion criteria: 1) Patient with bilateral cleft lip.

2) Patients with associated syndromes. 3) Patients with facial cleft. 4) Patients with previous lip repair.

\section{Ethical consideration:}

The protocol of the study is discussed and approved for clinical study by the Ethical Research Committee of Al-Azhar University. The procedures and the aim of the study were clearly explained to the patient and the family ${ }^{(7,8)}$. A written detailed informed consent is obtained before enrollment into the study. All patients were subjected to nasal and alveolus defect measurement [Figure 3]:
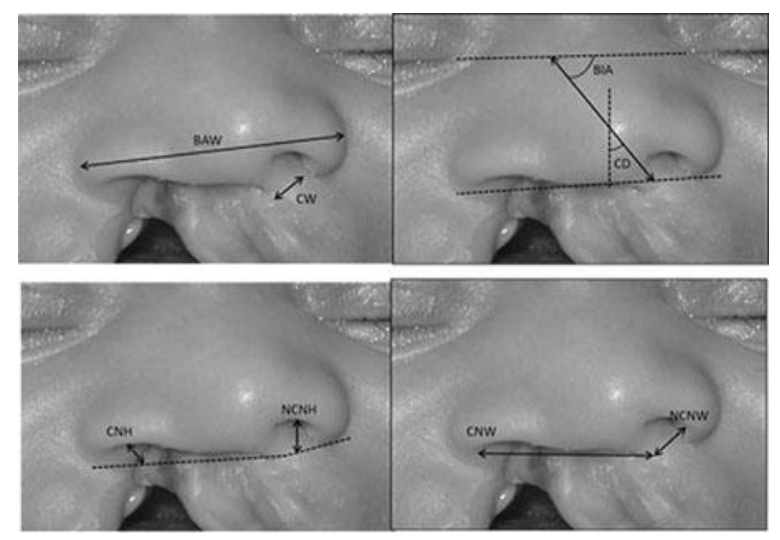

Figure 3: Diagram showing measurements. 


\section{NAM device formation:}

The device is similar to Grayson device but with modification in the oral part which formed from two parts with screw in the center between the two parts which allow narrowing of the oral part every other day, and this is cost effective and comfortable for the family. We teach the family how to deal with the device, especially its insertion and removal, and its cleaning at home for good hygiene. We adjust the oral part of the device every other day by narrowing the screw to reduce the alveolar defect. We adjust the nasal part of the device to maintain elevation of the depressed nostril every week.

When the alveolar defect reaches less than $0.6 \mathrm{~mm}$, we stopped the narrowing (molding) of the oral part and maintain nasal molding for 2 to 3 months.

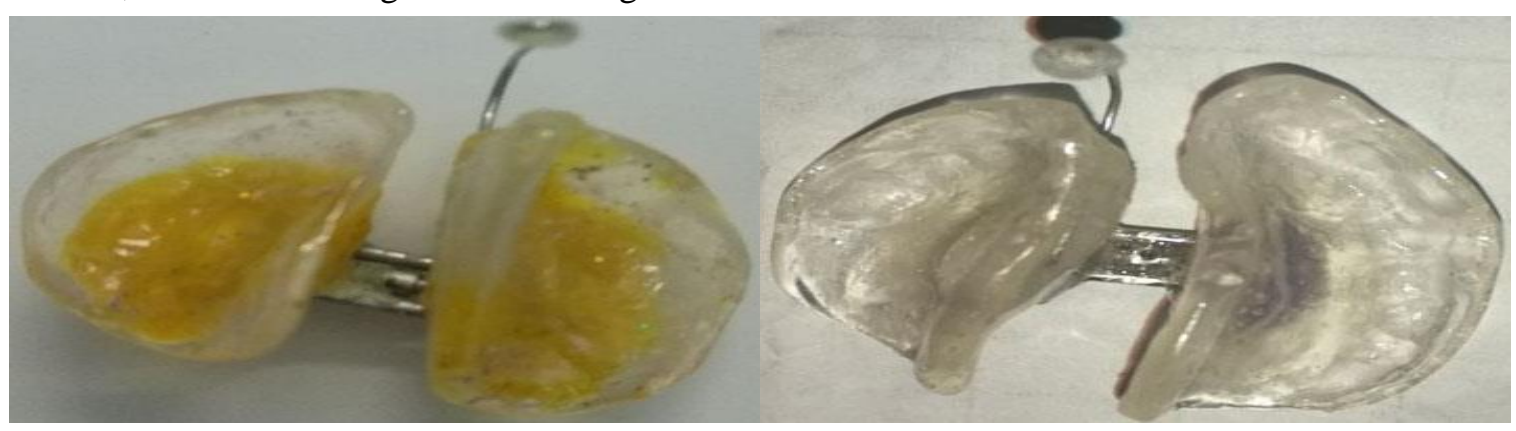

Figure 4: Photos of our nasoalveolar device.

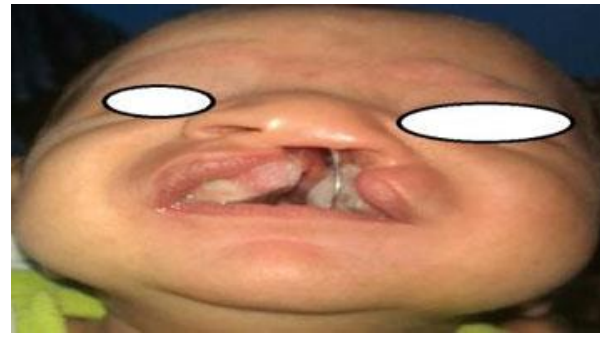

Figure 5: Photos of cases of nasoalveolar device.

After that measurements were taken again before the operation. All operations are done at Al-Azhar university hospitals (Al Hussein and Sayed Galal hospitals) in pediatric and plastic surgery departments under general anesthesia with oral central down endotracheal intubation in supine position with slightly extended head under complete a septic condition. The following steps are done:

All skin markings are made with either a methylene blue or marker.

The operation is started by identifying and marking the low point of Cupid's bow on the medial lip element.

The high point of Cupid's bow is then marked on the non-cleft side first and is typically 3-4 $\mathrm{mm}$ from Cupid's low point marking. We measure the distance from the low point of cupid's bow to the angle of the mouth in the non-cleft side by caliper. With the caliper the same distance from the other angel measured and we mark the cleft side. We put a point in the center of columella and from this point we draw a $\mathrm{C}$ shaped curve to the high point of Cupid's bow on the non-cleft side. We dissect the lips from periosteum of facial bone to the level of zygomatic arch in both sides to make freeing of lips and allow tension free closure of the cleft lip.

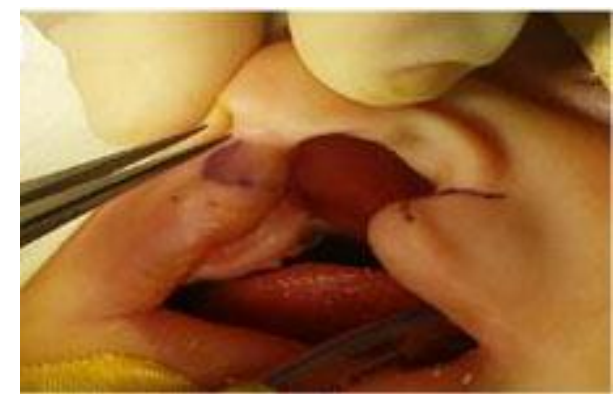

Figure 6: Preoperative skin markings in preparation

Then we take skin incision with scalpel NO. (15) starting from the lateral point of columella to the highest point Cupid's bow on the non-cleft side.

Then we take incision of $\mathrm{c}$ shaped curve starting from the center of columella to the highest point of Cupid's bow on the non-cleft side.

Then we make incision from the medial side of ala of the nose in the cleft site to the point of the lip in the same side and dissect the skin from the muscle and muscle from the mucosa to allow repair in 3 layers. 


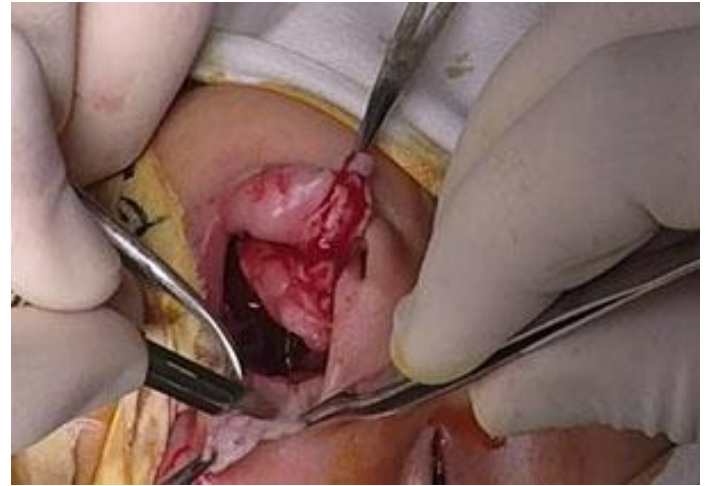

Figure 7: Dissection of muscle from skin and mucosa

After that we make a curve incision around ala of cleft side.

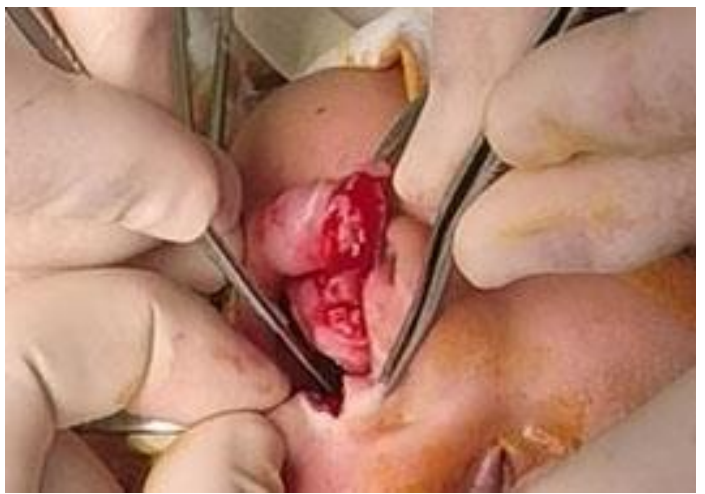

Figure 8: Subalar incision

We dissect the two cartilage of septum from each other and from the vomerine bone to allow freeing of septum.

Then we dissect the skin of columella from cartilage till the tip of the nose to release abnormal attachment between soft tissue and cartilage and dissect between the skin and lower lateral cartilage and dissect skin from cartilage till the septum to release abnormal attachment of skin to cartilage. Throughout the procedure good haemostasis is achieved. Then we start suturing the muscle using vicryl 4/0 with rounded needle by simple interrupted suture and suture the $\mathrm{C}$ shaped flap to the incision below the ala of the cleft side. After that, we construct the nasal floor with the c shaped flap, then we close the skin using prolene 5/0 and finally we realign the red line in the same level with simple interrupted suture and close the mucosa of the inner side of the lip with vicryl $4 / 0$ with rounded needle with simple interrupted suture.

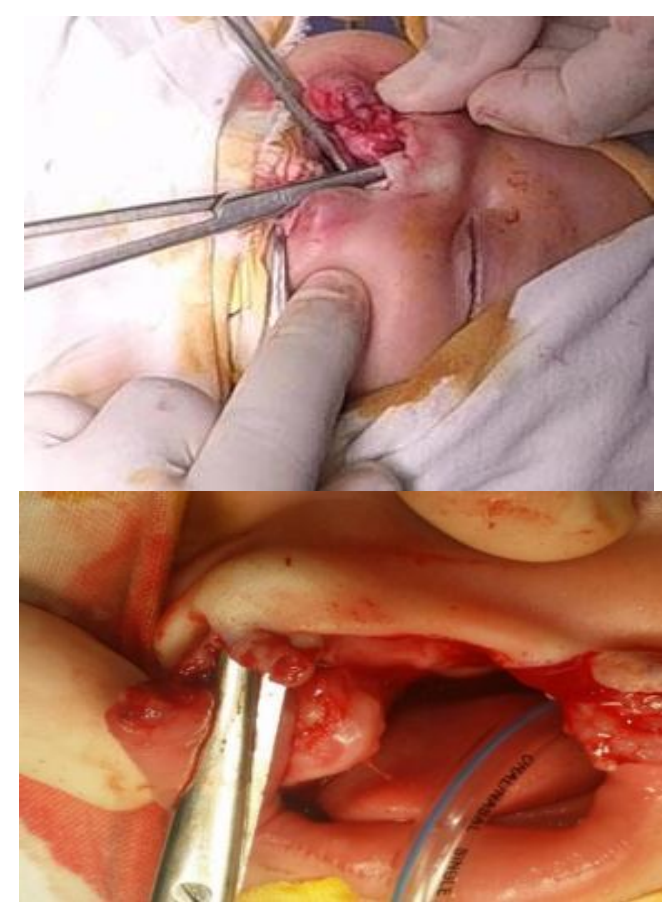

Figure 9: Dissection of skin from lower lateral cartilage.

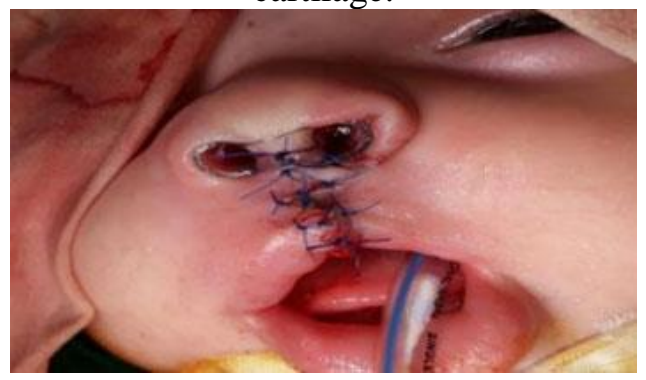

Figure 10 : Nasal stent

After that we insert a nasal stent which is handmade formed from nylton catheter 18 French. Then stristipe dressing is used to cover the wound. In the first postoperative day we remove wound cover and clean the wound, clean the nose from any discharge and apply dressing again. Daily dressing of the wound and cleaning the nose from any discharge is done. Removal of the stitch is done after 6 days postoperative. Nasal stent is left for 6 to 8 weeks postoperative until complete healing to avoid nasal stricture.The patients are closely followed up every one week for 3 months.

\section{Results}

From March 2016 to May 2018, thirty children 24 males and 6 females with male to female ratio $4: 1$, 16 Left side and 14 Right side with ratio 1.14:1 presented with complete cleft lip were referred to the pediatric surgery clinic, Al-Azhar University Hospitals. Mean age at presentation was $89.17 \pm$ 73.02 days (range, 15-260 days). Presurgical nasoalveolar molding was done for all patients with mean duration of molding 78.48 5.635 days 
(range, 66-87 days). There was highly significance statistical decrease of bialar width after use of nasoalveolar device and after operation from (3.217 \pm 0.5943$)$ before molding to $(2.897 \pm$ $0.5169)$ after molding to $(2.430 \pm 0.3120)$ [P value $<0.0001]$. There was significant increase in the columellar width as compared before and after molding [ $\mathrm{P}$ value $=0.01]$, high significant increase in columellar width as compared before molding and after operation [P value $=0.0089]$ and no significant increase in the width after molding and after operation $[\mathrm{P}$ value $=0.5725]$.

There was highly significant statistical increase in the none cleft columella height as compared before and after molding $[\mathrm{P}$ value $=0.0014]$, highly significant increase in none cleft columella height as compared before molding and after operation [P value $=0.0002]$ and no significant increase in the height after molding and after operation $[\mathrm{P}$ value $=$ 0.0831]. There was high significant increase in the cleft columella height as compared before and after molding $[\mathrm{P}$ value $=0.0001]$, high significant increase in cleft columella height as compared before molding and after operation $[\mathrm{P}$ value $=$ 0.0001] and high significant increase in the height after molding and after operation $[\mathrm{P}$ value $=$ $0.0001]$. There was increase in the cleft nostril height from $(0.07333 \pm 0.05833)$ before molding to $(0.2333 \pm 0.06065)$ after molding to $(0.4400 \pm 0.09685)$ after operation with highly significant statistical increase [P value $<0.0001]$. (Table 1), (Figure 1-12).

Table (1): Comparison between alveolar gap width before and after molding and after operation.

\begin{tabular}{|l|l|l|l|l|}
\hline Alveolar defect & Mean $\pm \mathrm{SD}$ & $\mathrm{P}$ & $\mathrm{P}$ & $\mathrm{P}$ \\
\multicolumn{1}{|c|}{} & & $\mathrm{T}_{1}$ & $\mathrm{~T}_{2}$ & $\mathrm{~T}_{3}$ \\
\hline Before molding & $\mathbf{1 . 2 1 3} \pm \mathbf{0 . 3 8 7 5}$ & $<\mathbf{0 . 0 0 0 1}$ & $<\mathbf{0 . 0 0 0 1}$ & $\mathbf{0 . 1 6 0 8}$ \\
& & $\mathbf{1 0 . 9 2}$ & $\mathbf{1 1 . 2 3}$ & $\mathbf{1 . 4 3 9}$ \\
\hline After molding & $\mathbf{0 . 5 6 0 0 \pm 0 . 1 2 7 6}$ & & & \\
\hline After operation & $\mathbf{0 . 5 4 6 7 \pm 0 . 1 3 3 2}$ & & & \\
& & & & \\
\hline
\end{tabular}

There was highly significant statistical decrease as compered between before and after molding and as compered before molding and after operation [P value $<0.0001]$ and no significant statistical decrease as compered between after molding and after operation $[\mathrm{P}$ value $=0.1608]$.

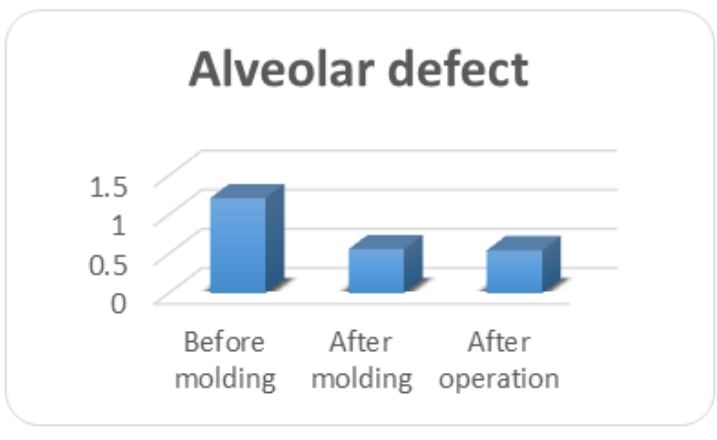

Figure 11: Comparison between alveolar defect gap before and after molding and after operation.

There was decrease in the angle of columella deviation from $(50.73 \pm 8.221)$ before molding to (25.23 \pm 6.393$)$ after molding to $(2.333 \pm 4.498)$ after operation with high significant statistical decrease [P value $<0.0001$.

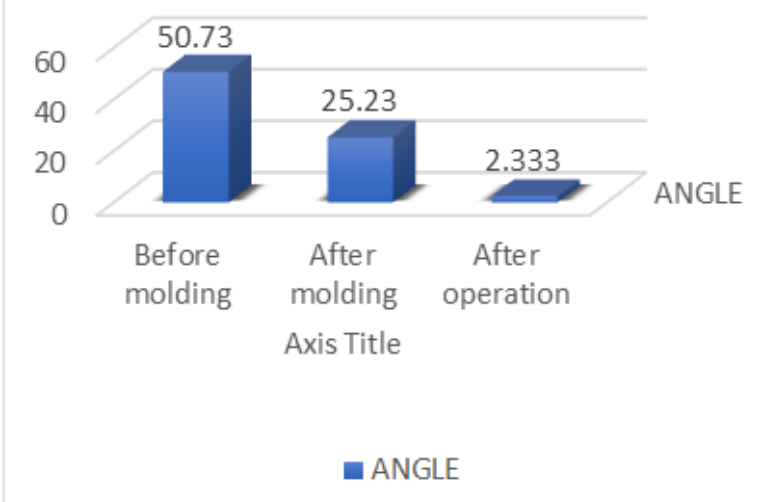

Figure 12: Comparison between degree of angle of deviations of columella before and after molding and after operation.

\section{Discussion}

The treatment of unilateral cleft lip, without exception, must involve correction of the nasal deformity. To obtain excellent results, the surgeon must integrate technique, teamwork, and timing based on multidisciplinary protocols developed over the years $\mathbf{( 9 , 1 0 , 1 1 )}^{\text {. }}$

NAM is an extension of alveolar molding that incorporates a nasal stent to support the nasal dome once the alveolar segments are aligned. Many studies have demonstrated improved pre-operative nose form ${ }^{(\mathbf{1 2}, 13,14)}$. However, long-term improvements are still unclear. Regarding the significant reduction in the alveolar defect after the use of NAM and growth of the cleft alveolus in the normal direction, this is similar to the study conducted by Shetty $\boldsymbol{e t}$ al. ${ }^{(24)}$ which found that the use of NAM improved the nasal projection, symmetry, and reduced the distance between alveolar segments. This made the nasolabial surgery easier for 
surgeons since the decrease in the distance between labial and alveolar segments eases tissues repair with a minimum of stress in the healing wound ${ }^{(15,16)}$. In this study the use of NAM lead to improvement in the nasal symmetry and decrease the size of the cleft defect which allow tension free repair of the lip and improve the result of primary rhinoplasty as regard symmetry of nostril size and decrease the angle of deviation of columella. In this study we use the nasal molding at the same time of alveolar molding and this similar to the result of Grayson ${ }^{(17)}$, Restrepo ${ }^{(18)}$ and Mishra ${ }^{\left({ }^{(19)}\right.}$ pioneers in PNAM, in their publication 1999, 2010, 2016 described that the nasal stent combined with the molding plate achieves nasal and alveolar symmetry, good projection of the nasal tip and concluded that surgical repair would no longer be a challenge. Another study carried out by Maull et al. ${ }^{(20)}$ similar to the one carried out in the Center of Medical Specialties of the State of Veracruz (CEMEV), concluded presurgical treatment with PNAM significantly decreases the asymmetry of the nose and the depressed nasal wing. In this study the use of NAM as early as 15 days after birth gives better result and this similar to the study published in by Mishra et al. ${ }^{(\mathbf{1 9})} 17$ patients with unilateral CLP showed an increase in the height of the wing of the nose with the use of PNAM and also demonstrated that it is more effective at early ages, maximum 6 weeks after birth. Liou et al. ${ }^{(21)}$ evaluated by means of photographs the changes that occurred in 25 patients with NAM and concluded that nasal asymmetry gradually improved significantly after the first surgery for primary closure of the lip or cheiloplasty and that a year after surgery, the changes remained stable and with good results. Although in this study stability was not investigated, we agree that presurgical treatment has excellent results in terms of nasal symmetry. Patel et al. ${ }^{(22)}$ estimated that PNAM and primary nasal reconstruction could lead to a saving of $\$ 491$ to $\$ 4893$ depending on the type of cleft when all associated costs were taken into consideration. Baek and $\operatorname{Son}^{(23)}$ carried out a three dimensional analysis of the effect of alveolar molding and suggested that the cleft gap was significantly reduced. It was also found that alveolar molding took place mainly in the anterior alveolar segment and growth occurred mainly in the posterior alveolar segment. Baek and Son ${ }^{(23)}$ reported that infant orthopedics does not have any influence on the maxillary arch dimensions. The timing of repair of the defect also plays an essential role. In this study all patients were done before the age of 5 months except only 3 patients were done after 5 months and all results were the same and there was no deferent in the outcome. A study conducted by Shetty et al. ${ }^{(24)}$ to evaluate the effect of NAM at different ages stated that favorable outcome was obtained when the treatment was initiated within 1-month of life; however, positive outcome was also achieved when the treatment was initiated within 5 months of life but to a lesser extent. In this study there was significant improvement in the nasal symmetry, decreased angular deviation of columella, elevate depressed ala of the nose, reduce the width of the cleft nostril, elongate the columellar length, and increase the height of the cleft nostril after the use of nasoalveolar molding and the molding facilitate the primary rhinoplasty and nasal measurements after operation were significantly improved. This similar to the studies conducted by Spengler $\boldsymbol{e t} \boldsymbol{a l} .{ }^{(25)}$ to assess the nasal changes after PNAM by evaluating the casts after NAM revealed that this therapy significantly improved the nasal symmetry. Columella deviation, length and width were also significantly improved. In this study we have 3 cases in which there was nostril stenosis and slightly depressed ala after operation and this was due to not use the nasal stent after the operation for sufficient time as the stent causing annoying of the family during insertion and removal. Similar results were reported by Pai $\boldsymbol{e t}$ al. ${ }^{(26)}$ who performed the evaluation based on the photographic analysis. However, some amount of relapse of the nostril width, height and angle of columella were observed at 1-year of age. However, early primary rhinoplasty procedures initially yielded good results, but due to the inherent dysmorphology of the nasal cartilages and due to the contractures after surgical repair, there was a return of original deformity. In this study we were use nasal molding at the same time of alveolar molding by adding of nasal stent to the device and adjust it to elevate the depressed ala of the cleft side and we didn't need the nasal floor to apply the stent as it connected to the oral part with wire. Grayson et al. ${ }^{(17)}$ added a nasal stent to presurgical alveolar molding plate, which did not require the presence of intact nasal floor and could exert a controlled force. In this study we stop alveolar molding and adjustment when alveolar defect reach $6 \mathrm{~mm}$ and continue on nasal molding. According to the Grayson technique, the most suitable moment occurs once the distance of the alveolar cleft is narrowed by alveolar molding to $5 \mathrm{~mm}$ or less. Commencement at this point and not earlier should avoid undesired lengthening of the alar rim as the initially highly stretched alar rim is more relaxed. In contrast, Figueroa and polly ${ }^{(27)}$ start alveolar and nasal molding simultaneously shortly after birth. When comparing the two techniques, Liao et al. ${ }^{(28)}$ describe that the nostril width is reduced significantly only in the Grayson group. In this study we use silicon nasal stent after operation for at least $6^{\text {th }}$ to $8^{\text {th }}$ weeks after operation and we learn the parents who to deal with it, who to insert it and who to remove it to clean. 
The postoperative use of nasal stents could be another positive factor that sustained the nasal shape, and its persistent use for 6 months was critical. This required parental cooperation. The gradual improvement in oral and nasal deformity during the course of NAM encourages the parents to collaborate with the treatment team, which is a key to our success. It is mandatory that parents are involved in and committed to the treatment in order to achieve the ideal results. Reasons for a temporary interruption of NAM or the premature termination of the treatment were either lack of parental support or child-related reasons such as skin irritations or restlessness. In this study we have two cases with postoperative lip notch and this attribute to improper alignment of the red line or improper dissection and suturing of the muscle near to the red line during the operation. This similar to results of the study done by Mbuyi-Musanzayi et al. ${ }^{(29)}$ which found that A notch on the lip was observed in $6.9 \%$ of the patients who had undergone surgery. The limitations of this study include, the small sample size and there was no control or comparative group for the technique, therefore, further controlled randomized studies are recommended, in order to draw a firm conclusion.

\section{Conclusion:}

Early use of nasoalveolar molding within fifteen days post-delivery is effective at reducing alveolar gap width (AGW), alignment of cleft alveolar segments, and improvement of nasal symmetry as long as the parents cooperate in placing and maintaining the nasoalveolar molding device in the newborn's mouth and nose. Primary rhinoplasty with cleft lip repair becomes easier since nasoalveolar molding diminishes the tension over the soft tissues and reduces severity of deformity in the lower lateral alar cartilages. Postoperative nasal stent has a great role in improve the esthetic outcome of primary rhinoplasty.

\section{References}

1. Calizolari E, Bianchi F, Rubini $\mathrm{M}$ et al. (2014): EUROCAT Working Group. Epidemiology of cleft in Europe: implications for genetic research. Cleft Palate Craniofac J., 41: 244-9.

2. Bhuskute AA and Tollefson TT (2016): Cleft Lip Repair, Nasoalveolar Molding, and Primary Cleft Rhinoplasty, Facial Plast Surg Clin N Am., 24: 453-466.

3. Liang Z, Yao J, Chen PK, Zheng C, Yang J (2017): Effect of presurgical nasoalveolar molding on nasal symmetry in unilateral complete cleft lip/palate patients after primary cheiloplasty without concomitant nasal cartilage dissection: Early childhood evaluation. The Cleft Palate-Craniofacial Journal, 14-296.

4. Gandedkar NH, Kiat CC, Kanesan P et al. (2015): Presurgical nasoalveolar molding therapy in cleft lip and palate individuals: Case series and review, APOS Trends Orthod., 5:208-14.

5. Singh DG, Bercowski DL, Santiago PE (2005): Three dimensional nasal changes following nasoalveolar molding in patients with unilateral cleft lip and palate: Geometric morphometrics. Cleft Palate Craniofac J., 42(4): 403-9.

6. Mulliken JB (2005): Double unilimb zplastic repair of microform cleft lip. Plast Reconstr Surg., 116:1623.

7. Sykes JM and Jang YJ (2009): Cleft lip rhinoplasty. Facial Plast Surg Clin North Am., 17(1):133-44, vii.

8. Shih CW and Sykes JM (2002): Correction of the cleft-lip nasal deformity. Fac Plast Surg., 18: 253-62.

9. Fisher MD, Fisher DM, Marcus JR (2014): Correction of the Cleft Nasal Deformity: From Infancy to Maturity, Clin Plastic Surg., 41: 283-299.

10. Tanikawa DYS, Alonso N, Rocha DL (2010): Evaluation of primary cleft nose repair. J Craniofac Surg., 21:1519-1524.

11. Byrd HS, El-Musa KA, Yazdani A (2007): Definitive repair of the unilateral cleft lip nasal deformity. Plast Reconstr Surg., 120:1348-1356.

12. Shetty V, Vyas HJ, Sharma SM et al. (2012): A Comparison of results using nasoalveolar molding in cleft infants treated within 1month of life versus those treated after this period: development of new protocol. Int $\mathbf{J}$ Oral Maxillofacial Surg., 41(1): 28-36.

13. Kaufman Y, Buchanan EP, Wolfswinkel EM et al. (2012): Cleft Nasal Deformity and Rhinoplasty, Semin Plast Surg., 26:184-190.

14. Ezzat C, Chavarria C, Teichgraeber J et al. (2007): Presurgical nasoalveolar molding therapy for the treatment of unilateral cleft lip and palate: Cleft Palate Craniofac J., 44(1): 8-12.

15. Vyloppilli S, Krishnakumar K S, Sayd S et al. (2017): Postoperative alar base 
symmetry in complete unilateral cleft lip and palate: A prospective study. Journal of Plastic, Reconstructive \& Aesthetic Surgery, 70(11): 1614- 1619.

16. Uzel A and Alparslan ZN (2011): Longterm effects of presurgical infant orthopedics in patients with cleft lip and palate: a systematic review. Cleft PalateCraniofacial J., 48(5):587-95.

17. Grayson B, Santiago $P$, Brecht $L$ et al. (1999): Presurgical nasoalveolar molding in patients with cleft lip and palate. Cleft Palate Craniofac J., 36: 486-498.

18. Restrepo NL, Carvallo JRH, Cruz JRG (2016): Efficacy of the nasal molding in patients with unilateral cleft lip and palate in newborn to 6-month-old patients. Revista Mexicana de Ortodoncia, 4(4):238-243.

19. Mishra B, Singh AK, Zaidi J et al. (2010): Presurgical nasoalveolar molding for correction of cleft lip nasal deformity: Experience from northern India. Plast Surg J., 10:443-57.

20. Maull D, Grayson B, Cutting Cet al. (1999): Long-term effects of nasoalveolar molding on three-dimensional nasal shape in unilateral clefts. Cleft Palate Craniofac J., 36: 391-397.

21. Liou E, Subramanian M, Chen $P$ et al. (2004): The progressive changes of nasal symmetry and growth after nasoalveolar molding: a three-year follow-up study. Plast Reconstr Surg., 114: 858-864.

22. Patel PA, Rubin MS, Clouston S et al. (2015): Comparative study of early secondary nasal revisions and costs in patients with clefts treated with and without nasoalveolar molding. J Craniofacial Surg., 26: 1229-33.
23. Baek SH and Son WS (2006): Difference in alveolar molding effect and growth in the cleft segments: 3 dimensional analyses of unilateral cleft lip and palate patients. Oral Surg Oral Med Oral Pathol Oral Radiol Endod., 102(2): 160.

24. Shetty V, Thakral A, Sreekumar C (2016): Comparison of early onset nasoalveolar molding with patients who presented for molding up to 1 year of age. J Oral Maxillofac Surg., 74:811-827

25. Spengler AL, Chavaria $C$, Teichgraeber JF et al. (2006): Pre surgical Nasoalveolar molding therapy for the treatment of bilateral cleft lip and palate: A preliminary study. Cleft Palate Craniofacial J., 43(3): 321-8.

26. Pai BCJ, Ko EWC, Huang CS et al. (2005): Symmetry of the nose after presurgical nasoalveolar molding in infants with unilateral cleft lip and palate: a preliminary study. The Cleft palatecraniofacial journal, 42(6): 658-663.

27. Figueroa AA and Polley JW (2006): Orthodontics in cleft lip and palate management. Plastic Surgery Vol 4, Pediatric Plastic Surgery 2nd ed. Philadelphia: Elsevier.

28. Liao YF, Hsieh YJ, Chen IJ et al. (2012): Comparative outcomes of two nasoalveolar molding techniques for unilateral cleft nose deformity. Plast Reconstr Surg., 130: 1289-1295.

29. Mbuyi-Musanzayi S, Katombe FT, Tshilobo PL et al. (2017): Anthropometric and aesthetic outcomes for the nasolabial region in 101 consecutive African children with unilateral cleft lip one year after repair using the anatomical subunit approximation technique. Int journal of oral and maxillofacial surgery, 46 (10): 1338 -1345. 